\title{
The Shrinking Projection Method for Common Solutions of Generalized Mixed Equilibrium Problems and Fixed Point Problems for Strictly Pseudocontractive Mappings
}

\author{
Thanyarat Jitpeera and Poom Kumam \\ Department of Mathematics, Faculty of Science, King Mongkut's University of Technology Thonburi \\ (KMUTT), Bangmod, Bangkok 10140, Thailand
}

Correspondence should be addressed to Poom Kumam, poom.kum@kmutt.ac.th

Received 21 September 2010; Revised 14 December 2010; Accepted 20 January 2011

Academic Editor: Jewgeni Dshalalow

Copyright (C) 2011 T. Jitpeera and P. Kumam. This is an open access article distributed under the Creative Commons Attribution License, which permits unrestricted use, distribution, and reproduction in any medium, provided the original work is properly cited.

\begin{abstract}
We introduce the shrinking hybrid projection method for finding a common element of the set of fixed points of strictly pseudocontractive mappings, the set of common solutions of the variational inequalities with inverse-strongly monotone mappings, and the set of common solutions of generalized mixed equilibrium problems in Hilbert spaces. Furthermore, we prove strong convergence theorems for a new shrinking hybrid projection method under some mild conditions. Finally, we apply our results to Convex Feasibility Problems (CFP). The results obtained in this paper improve and extend the corresponding results announced by Kim et al. (2010) and the previously known results.
\end{abstract}

\section{Introduction}

Let $H$ be a real Hilbert space with inner product $\langle\cdot, \cdot\rangle$ and norm $\|\cdot\|$, and let $E$ be a nonempty closed convex subset of $H$. Let $T: E \rightarrow E$ be a mapping. In the sequel, we will use $F(T)$ to denote the set of fixed points of $T$, that is, $F(T)=\{x \in E: T x=x\}$. We denote weak convergence and strong convergence by notations $\rightarrow$ and $\rightarrow$, respectively.

Let $S: E \rightarrow E$ be a mapping. Then $S$ is called

(1) nonexpansive if

$$
\|S x-S y\| \leq\|x-y\|, \quad \forall x, y \in E,
$$


(2) strictly pseudocontractive with the coefficient $k \in[0,1)$ if

$$
\|S x-S y\|^{2} \leq\|x-y\|^{2}+k\|(I-S) x-(I-S) y\|^{2}, \quad \forall x, y \in E,
$$

(3) pseudocontractive if

$$
\|S x-S y\|^{2} \leq\|x-y\|^{2}+\|(I-S) x-(I-S) y\|^{2}, \quad \forall x, y \in E .
$$

The class of strictly pseudocontractive mappings falls into the one between classes of nonexpansive mappings and pseudocontractive mappings. Within the past several decades, many authors have been devoted to the studies on the existence and convergence of fixed points for strictly pseudocontractive mappings. In 2008, Zhou [1] considered a convex combination method to study strictly pseudocontractive mappings. More precisely, take $k \in[0,1)$, and define a mapping $S_{k}$ by

$$
S_{k} x=k x+(1-k) S x, \quad \forall x \in E,
$$

where $S$ is strictly pseudocontractive mappings. Under appropriate restrictions on $k$, it is proved that the mapping $S_{k}$ is nonexpansive. Therefore, the techniques of studying nonexpansive mappings can be applied to study more general strictly pseudocontractive mappings.

Recall that letting $A: E \rightarrow H$ be a mapping, then $A$ is called

(1) monotone if

$$
\langle A x-A y, x-y\rangle \geq 0, \quad \forall x, y \in E
$$

(2) $\beta$-inverse-strongly monotone if there exists a constant $\beta>0$ such that

$$
\langle A x-A y, x-y\rangle \geq \beta\|A x-A y\|^{2}, \quad \forall x, y \in E .
$$

The domain of the function $\varphi: E \rightarrow \mathbb{R} \cup\{+\infty\}$ is the set $\operatorname{dom} \varphi=\{x \in E: \varphi(x)<+\infty\}$. Let $\varphi: E \rightarrow \mathbb{R} \cup\{+\infty\}$ be a proper extended real-valued function and let $F$ be a bifunction of $E \times E$ into $\mathbb{R}$ such that $E \cap \operatorname{dom} \varphi \neq \emptyset$, where $\mathbb{R}$ is the set of real numbers.

There exists the generalized mixed equilibrium problem for finding $x \in E$ such that

$$
F(x, y)+\langle A x, y-x\rangle+\varphi(y)-\varphi(x) \geq 0, \quad \forall y \in E
$$

The set of solutions of (1.7) is denoted by $\operatorname{GMEP}(F, \varphi, A)$, that is,

$$
\operatorname{GMEP}(F, \varphi, A)=\{x \in E: F(x, y)+\langle A x, y-x\rangle+\varphi(y)-\varphi(x) \geq 0, \forall y \in E\}
$$


We see that $x$ is a solution of a problem (1.7) which implies that $x \in \operatorname{dom} \varphi=\{x \in E: \varphi(x)<$ $+\infty\}$.

In particular, if $A \equiv 0$, then the problem (1.7) is reduced into the mixed equilibrium problem [2] for finding $x \in E$ such that

$$
F(x, y)+\varphi(y)-\varphi(x) \geq 0, \quad \forall y \in E .
$$

The set of solutions of (1.9) is denoted by $\operatorname{MEP}(F, \varphi)$.

If $A \equiv 0$ and $\varphi \equiv 0$, then the problem (1.7) is reduced into the equilibrium problem [3] for finding $x \in E$ such that

$$
F(x, y) \geq 0, \quad \forall y \in E
$$

The set of solutions of (1.10) is denoted by $\mathrm{EP}(F)$. This problem contains fixed point problems and includes as special cases numerous problems in physics, optimization, and economics. Some methods have been proposed to solve the equilibrium problem; please consult $[4,5]$.

If $F \equiv 0$ and $\varphi \equiv 0$, then the problem (1.7) is reduced into the Hartmann-Stampacchia variational inequality [6] for finding $x \in E$ such that

$$
\langle A x, y-x\rangle \geq 0, \quad \forall y \in E
$$

The set of solutions of (1.11) is denoted by $\operatorname{VI}(E, A)$. The variational inequality has been extensively studied in the literature. See, for example, [7-10] and the references therein.

Many authors solved the problems $\operatorname{GMEP}(F, \varphi, A), \operatorname{MEP}(F, \varphi)$, and $\operatorname{EP}(F)$ based on iterative methods; see, for instance, $[4,5,11-25]$ and reference therein.

In 2007, Tada and Takahashi [26] introduced a hybrid method for finding the common element of the set of fixed point of nonexpansive mapping and the set of solutions of equilibrium problems in Hilbert spaces. Let $\left\{x_{n}\right\}$ and $\left\{u_{n}\right\}$ be sequences generated by the following iterative algorithm:

$$
\begin{gathered}
x_{1}=x \in H, \\
F\left(u_{n}, y\right)+\frac{1}{r_{n}}\left\langle y-u_{n}, u_{n}-x_{n}\right\rangle \geq 0, \quad \forall y \in E, \\
w_{n}=\left(1-\alpha_{n}\right) x_{n}+\alpha_{n} S u_{n}, \\
E_{n}=\left\{z \in H:\left\|w_{n}-z\right\| \leq\left\|x_{n}-z\right\|\right\}, \\
D_{n}=\left\{z \in H:\left\langle x_{n}-z, x-x_{n}\right\rangle \geq 0\right\}, \\
x_{n+1}=P_{E_{n} \cap D_{n}} x, \quad \forall n \geq 1 .
\end{gathered}
$$

Then, they proved that, under certain appropriate conditions imposed on $\left\{\alpha_{n}\right\}$ and $\left\{r_{n}\right\}$, the sequence $\left\{x_{n}\right\}$ generated by (1.12) converges strongly to $P_{F(S) \cap E P(F)} x$.

In 2009, Qin and Kang [27] introduced an explicit viscosity approximation method for finding a common element of the set of fixed point of strictly pseudocontractive mappings 
and the set of solutions of variational inequalities with inverse-strongly monotone mappings in Hilbert spaces:

$$
\begin{gathered}
x_{1} \in E, \\
z_{n}=P_{E}\left(x_{n}-\mu_{n} C x_{n}\right), \\
y_{n}=P_{E}\left(x_{n}-\lambda_{n} B x_{n}\right), \\
x_{n+1}=\epsilon_{n} f\left(x_{n}\right)+\beta_{n} x_{n}+\gamma_{n}\left[\alpha_{n}^{(1)} S_{k} x_{n}+\alpha_{n}^{(2)} y_{n}+\alpha_{n}^{(3)} z_{n}\right], \quad \forall n \geq 1 .
\end{gathered}
$$

Then, they proved that, under certain appropriate conditions imposed on $\left\{\epsilon_{n}\right\},\left\{\beta_{n}\right\},\left\{\gamma_{n}\right\}$, $\left\{\alpha_{n}^{(1)}\right\},\left\{\alpha_{n}^{(2)}\right\}$, and $\left\{\alpha_{n}^{(3)}\right\}$, the sequence $\left\{x_{n}\right\}$ generated by (1.13) converges strongly to $q \in$ $F(S) \cap \operatorname{VI}(E, B) \cap \operatorname{VI}(E, C)$, where $q=P_{F(S) \cap V I}(E, B) \cap V I(E, C) f(q)$.

In 2010, Kumam and Jaiboon [28] introduced a new method for finding a common element of the set of fixed point of strictly pseudocontractive mappings, the set of common solutions of variational inequalities with inverse-strongly monotone mappings, and the set of common solutions of a system of generalized mixed equilibrium problems in Hilbert spaces. Then, they proved that, under certain appropriate conditions imposed on $\left\{\epsilon_{n}\right\},\left\{\beta_{n}\right\}$, and $\left\{\alpha_{n}^{(i)}\right\}$, where $i=1,2,3,4,5$. The sequence $\left\{x_{n}\right\}$ converges strongly to $q \in \Theta:=F(S) \cap \operatorname{VI}(E, B) \cap$ $\operatorname{VI}(E, C) \cap \operatorname{GMEP}\left(F_{1}, \varphi, A_{1}\right) \cap \operatorname{GMEP}\left(F_{2}, \varphi, A_{2}\right)$, where $q=P_{\Theta}(I-A+\gamma f)(q)$.

In this paper, motivate, by Tada and Takahashi [26], Qin and Kang [27], and Kumam and Jaiboon [28], we introduce a new shrinking projection method for finding a common element of the set of fixed points of strictly pseudocontractive mappings, the set of common solutions of generalized mixed equilibrium problems, and the set of common solutions of the variational inequalities for inverse-strongly monotone mappings in Hilbert spaces. Finally, we apply our results to Convex Feasibility Problems (CFP). The results obtained in this paper improve and extend the corresponding results announced by the previously known results.

\section{Preliminaries}

Let $H$ be a real Hilbert space, and let $E$ be a nonempty closed convex subset of $H$. In a real Hilbert space $H$, it is well known that

$$
\|\lambda x+(1-\lambda) y\|^{2}=\lambda\|x\|^{2}+(1-\lambda)\|y\|^{2}-\lambda(1-\lambda)\|x-y\|^{2},
$$

for all $x, y \in H$ and $\lambda \in[0,1]$.

For any $x \in H$, there exists a unique nearest point in $E$, denoted by $P_{E} x$, such that

$$
\left\|x-P_{E} x\right\| \leq\|x-y\|, \quad \forall y \in E
$$

The mapping $P_{E}$ is called the metric projection of $H$ onto $E$.

It is well known that $P_{E}$ is a firmly nonexpansive mapping of $H$ onto $E$, that is,

$$
\left\langle x-y, P_{E} x-P_{E} y\right\rangle \geq\left\|P_{E} x-P_{E} y\right\|^{2}, \quad \forall x, y \in H
$$


Moreover, $P_{E} x$ is characterized by the following properties: $P_{E} x \in E$ and

$$
\begin{gathered}
\left\langle x-P_{E} x, y-P_{E} x\right\rangle \leq 0, \\
\|x-y\|^{2} \geq\left\|x-P_{E} x\right\|^{2}+\left\|y-P_{E} x\right\|^{2}
\end{gathered}
$$

for all $x \in H, y \in E$.

Lemma 2.1. Let $E$ be a nonempty closed convex subset of a real Hilbert space $H$. Given $x \in H$ and $z \in E$, then,

$$
z=P_{E} x \Longleftrightarrow\langle x-z, y-z\rangle \leq 0, \quad \forall y \in E .
$$

Lemma 2.2. Let $H$ be a Hilbert space, let $E$ be a nonempty closed convex subset of $H$, and let $B$ be a mapping of $E$ into $H$. Let $u \in E$. Then, for $\lambda>0$,

$$
u \in \mathrm{VI}(E, B) \Longleftrightarrow u=P_{E}(u-\lambda B u),
$$

where $P_{E}$ is the metric projection of $H$ onto $E$.

Lemma 2.3 (see [1]). Let $E$ be a nonempty closed convex subset of a real Hilbert space $H$, and let $S: E \rightarrow E$ be a $k$-strictly pseudocontractive mapping with a fixed point. Then $F(S)$ is closed and convex. Define $S_{k}: E \rightarrow E$ by $S_{k}=k x+(1-k) S x$ for each $x \in E$. Then $S_{k}$ is nonexpansive such that $F\left(S_{k}\right)=F(S)$.

Lemma 2.4 (see [29]). Let $E$ be a closed convex subset of a real Hilbert space $H$, and let $S: E \rightarrow E$ be a nonexpansive mapping. Then $I-S$ is demiclosed at zero; that is,

$$
x_{n} \rightarrow x, \quad x_{n}-S x_{n} \longrightarrow 0
$$

implies $x=S x$.

Lemma 2.5 (see [30]). Each Hilbert space $H$ satisfies the Kadec-Klee property, for any sequence $\left\{x_{n}\right\}$ with $x_{n} \rightarrow x$ and $\left\|x_{n}\right\| \rightarrow\|x\|$ together implying $\left\|x_{n}-x\right\| \rightarrow 0$.

Lemma 2.6 (see [31]). Let $E$ be a closed convex subset of $H$. Let $\left\{x_{n}\right\}$ be a bounded sequence in $H$. Assume that

(1) the weak $\omega$-limit set $\omega_{w}\left(x_{n}\right) \subset E$,

(2) for each $z \in E, \lim _{n \rightarrow \infty}\left\|x_{n}-z\right\|$ exists.

Then $\left\{x_{n}\right\}$ is weakly convergent to a point in $E$.

Lemma 2.7 (see [32]). Let $E$ be a closed convex subset of $H$. Let $\left\{x_{n}\right\}$ be a sequence in $H$ and $u \in H$. Let $q=P_{E} u$. If $\left\{x_{n}\right\}$ is $\omega_{w}\left(x_{n}\right) \subset E$ and satisfies the condition

$$
\left\|x_{n}-u\right\| \leq\|u-q\|
$$

for all $n$, then $x_{n} \rightarrow q$. 
Lemma 2.8 (see [33]). Let $E$ be a nonempty closed convex subset of a strictly convex Banach space $X$. Let $\left\{T_{n}: n \in \mathbb{N}\right\}$ be a sequence of nonexpansive mappings on E. Suppose $\bigcap_{n=1}^{\infty} F\left(T_{n}\right)$ is nonempty. Let $\delta_{n}$ be a sequence of positive number with $\sum_{n=1}^{\infty} \delta_{n}=1$. Then a mapping $S$ on E defined by

$$
S x=\sum_{n=1}^{\infty} \delta_{n} T_{n} x
$$

for $x \in E$ is well defined, nonexpansive, and $F(S)=\bigcap_{n=1}^{\infty} F\left(T_{n}\right)$ holds.

For solving the mixed equilibrium problem, let us give the following assumptions for the bifunction $F$, the function $A$, and the set $E$ :

(A1) $F(x, x)=0$ for all $x \in E$

(A2) $F$ is monotone, that is, $F(x, y)+F(y, x) \leq 0$ for all $x, y \in E$

(A3) for each $x, y, z \in E, \lim _{t \rightarrow 0} F(t z+(1-t) x, y) \leq F(x, y)$

(A4) for each $x \in E, y \mapsto F(x, y)$ is convex and lower semicontinuous

(A5) for each $y \in E, x \mapsto F(x, y)$ is weakly upper semicontinuous

(B1) for each $x \in H$ and $r>0$, there exists a bounded subset $D_{x} \subseteq E$ and $y_{x} \in E$ such that, for any $z \in E \backslash D_{x}$,

$$
F\left(z, y_{x}\right)+\varphi\left(y_{x}\right)-\varphi(z)+\frac{1}{r}\left\langle y_{x}-z, z-x\right\rangle<0,
$$

(B2) $E$ is a bounded set.

By similar argument as in the proof of Lemma 2.9 in [34], we have the following lemma appearing.

Lemma 2.9. Let $E$ be a nonempty closed convex subset of $H$. Let $F: E \times E \rightarrow \mathbb{R}$ be a bifunction that satisfies (A1)-(A5), and let $\varphi: E \rightarrow \mathbb{R} \cup\{+\infty\}$ be a proper lower semicontinuous and convex function. Assume that either (B1) or (B2) holds. For $r>0$ and $x \in H$, define a mapping $T_{r}^{F}: H \rightarrow E$ as follows:

$$
T_{r}^{F}(x)=\left\{z \in E: F(z, y)+\varphi(y)-\varphi(z)+\frac{1}{r}\langle y-z, z-x\rangle \geq 0, \forall y \in E\right\}
$$

for all $z \in H$. Then, the following hold:

(1) for each $x \in H, T_{r}^{F}(x) \neq \emptyset$,

(2) $T_{r}^{F}$ is single valued,

(3) $T_{r}^{F}$ is firmly nonexpansive, that is, for any $x, y \in H$,

$$
\left\|T_{r}^{F} x-T_{r}^{F} y\right\|^{2} \leq\left\langle T_{r}^{F} x-T_{r}^{F} y, x-y\right\rangle
$$

(4) $F\left(T_{r}^{F}\right)=\operatorname{MEP}(F, \varphi)$,

(5) $\operatorname{MEP}(F, \varphi)$ is closed and convex. 
Lemma 2.10. Let $H$ be a Hilbert space, let $E$ be a nonempty closed convex subset of $H$, and let $A: E \rightarrow H$ be $\rho$-inverse-strongly monotone. If $0<r \leq 2 \rho$, then $I-\rho A$ is a nonexpansive mapping in $H$.

Proof. For all $x, y \in E$ and $0<r \leq 2 \rho$, we have

$$
\begin{aligned}
\|(I-r A) x-(I-r A) y\|^{2} & =\|(x-y)-r(A x-A y)\|^{2} \\
& =\|x-y\|^{2}-2 r\langle x-y, A x-A y\rangle+r^{2}\|A x-A y\|^{2} \\
& \leq\|x-y\|^{2}-2 r \rho\|A x-A y\|+r^{2}\|A x-A y\|^{2} \\
& =\|x-y\|^{2}+r(r-2 \rho)\|A x-A y\|^{2} \\
& \leq\|x-y\|^{2} .
\end{aligned}
$$

So, $I-\rho A$ is a nonexpansive mapping of $E$ into $H$.

\section{Main Results}

In this section, we prove a strong convergence theorem of the new shrinking projection method for finding a common element of the set of fixed points of strictly pseudocontractive mappings, the set of common solutions of generalized mixed equilibrium problems and the set of common solutions of the variational inequalities with inverse-strongly monotone mappings in Hilbert spaces.

Theorem 3.1. Let $E$ be a nonempty closed convex subset of a real Hilbert space $H$. Let $F_{1}$ and $F_{2}$ be two bifunctions from $E \times E$ to $\mathbb{R}$ satisfying (A1)-(A5), and let $\varphi: E \rightarrow \mathbb{R} \cup\{+\infty\}$ be a proper lower semicontinuous and convex function with either (B1) or (B2). Let $A_{1}, A_{2}, B, C$ be four $\rho, \omega$, $\beta$, $\xi$-inverse-strongly monotone mappings of $E$ into $H$, respectively. Let $S: E \rightarrow E$ be a $k$-strictly pseudocontractive mapping with a fixed point. Define a mapping $S_{k}: E \rightarrow E$ by $S_{k} x=k x+(1-k) S x$, for all $x \in$ E. Suppose that

$$
\Theta:=F(S) \cap \operatorname{GMEP}\left(F_{1}, \varphi, A_{1}\right) \cap \operatorname{GMEP}\left(F_{2}, \varphi, A_{2}\right) \cap \operatorname{VI}(E, B) \cap \operatorname{VI}(E, C) \neq \emptyset .
$$

Let $\left\{x_{n}\right\}$ be a sequence generated by the following iterative algorithm:

$$
\begin{gathered}
x_{0} \in H, \quad E_{1}=E, \quad x_{1}=P_{E_{1}} x_{0}, \quad u_{n} \in E, \quad v_{n} \in E, \\
F_{1}\left(u_{n}, u\right)+\varphi(u)-\varphi\left(u_{n}\right)+\left\langle A_{1} x_{n}, u-u_{n}\right\rangle+\frac{1}{r_{n}}\left\langle u-u_{n}, u_{n}-x_{n}\right\rangle \geq 0, \quad \forall u \in E, \\
F_{2}\left(v_{n}, v\right)+\varphi(v)-\varphi\left(v_{n}\right)+\left\langle A_{2} x_{n}, v-v_{n}\right\rangle+\frac{1}{s_{n}}\left\langle v-v_{n}, v_{n}-x_{n}\right\rangle \geq 0, \quad \forall v \in E, \\
y_{n}=P_{E}\left(x_{n}-\lambda_{n} B x_{n}\right), \quad z_{n}=P_{E}\left(x_{n}-\mu_{n} C x_{n}\right),
\end{gathered}
$$




$$
\begin{gathered}
t_{n}=\alpha_{n}^{(1)} S_{k} x_{n}+\alpha_{n}^{(2)} y_{n}+\alpha_{n}^{(3)} z_{n}+\alpha_{n}^{(4)} u_{n}+\alpha_{n}^{(5)} v_{n}, \\
E_{n+1}=\left\{w \in E_{n}:\left\|t_{n}-w\right\| \leq\left\|x_{n}-w\right\|\right\}, \\
x_{n+1}=P_{E_{n+1}} x_{0}, \quad \forall n \geq 0
\end{gathered}
$$

where $\left\{\alpha_{n}^{(i)}\right\}$ are sequences in $(0,1)$, where $i=1,2,3,4,5, r_{n} \in(0,2 \rho), s_{n} \in(0,2 \omega)$, and $\left\{\lambda_{n}\right\},\left\{\mu_{n}\right\}$ are positive sequences. Assume that the control sequences satisfy the following restrictions:

(C1) $\sum_{i=1}^{5} \alpha_{n}^{(i)}=1$,

(C2) $\lim _{n \rightarrow \infty} \alpha_{n}^{(i)}=\alpha^{(i)} \in(0,1)$, where $i=1,2,3,4,5$,

(C3) $a \leq r_{n} \leq 2 \rho$ and $b \leq s_{n} \leq 2 \omega$, where $a, b$ are two positive constants,

(C4) $c \leq \lambda_{n} \leq 2 \beta$ and $d \leq \mu_{n} \leq 2 \xi$, where $c$, $d$ are two positive constants,

(C5) $\lim _{n \rightarrow \infty}\left|\lambda_{n+1}-\lambda_{n}\right|=\lim _{n \rightarrow \infty}\left|\mu_{n+1}-\mu_{n}\right|=0$.

Then, $\left\{x_{n}\right\}$ converges strongly to $P_{\Theta} x_{0}$.

Proof. Letting $p \in \Theta$ and by Lemma 2.9, we obtain

$$
p=P_{E}\left(p-\lambda_{n} B p\right)=P_{E}\left(p-\mu_{n} C p\right)=T_{r_{n}}^{F_{1}}\left(I-r_{n} A_{1}\right) p=T_{s_{n}}^{F_{2}}\left(I-s_{n} A_{2}\right) p .
$$

Note that $u_{n}=T_{r_{n}}^{F_{1}}\left(I-r_{n} A_{1}\right) x_{n} \in \operatorname{dom} \varphi$ and $v_{n}=T_{s_{n}}^{F_{2}}\left(I-s_{n} A_{2}\right) x_{n} \in \operatorname{dom} \varphi$, then we have

$$
\begin{gathered}
\left\|u_{n}-p\right\|=\left\|T_{r_{n}}^{F_{1}}\left(I-r_{n} A_{1}\right) x_{n}-T_{r_{n}}^{F_{1}}\left(I-r_{n} A_{1}\right) p\right\| \leq\left\|x_{n}-p\right\|, \\
\left\|v_{n}-p\right\|=\left\|T_{s_{n}}^{F_{2}}\left(I-s_{n} A_{2}\right) x_{n}-T_{s_{n}}^{F_{2}}\left(I-s_{n} A_{2}\right) p\right\| \leq\left\|x_{n}-p\right\| .
\end{gathered}
$$

Next, we will divide the proof into six steps.

Step 1. We show that $\left\{x_{n}\right\}$ is well defined and $E_{n}$ is closed and convex for any $n \geq 1$.

From the assumption, we see that $E_{1}=E$ is closed and convex. Suppose that $E_{k}$ is closed and convex for some $k \geq 1$. Next, we show that $E_{k+1}$ is closed and convex for some $k$. For any $p \in E_{k}$, we obtain

$$
\left\|t_{k}-p\right\| \leq\left\|x_{k}-p\right\|
$$

is equivalent to

$$
\left\|t_{k}-p\right\|^{2}+2\left\langle t_{k}-x_{k}, x_{k}-p\right\rangle \leq 0
$$

Thus, $E_{k+1}$ is closed and convex. Then, $E_{n}$ is closed and convex for any $n \geq 1$. This implies that $\left\{x_{n}\right\}$ is well defined. 
Step 2. We show that $\Theta \subset E_{n}$ for each $n \geq 1$. From the assumption, we see that $\Theta \subset E=E_{1}$. Suppose $\Theta \subset E_{k}$ for some $k \geq 1$. For any $p \in \Theta \subset E_{k}$, since $y_{n}=P_{E}\left(x_{n}-\lambda_{n} B x_{n}\right)$ and $z_{n}=P_{E}\left(x_{n}-\mu_{n} C x_{n}\right)$, for each $\lambda_{n} \leq 2 \beta$ and $\mu_{n} \leq 2 \xi$ by Lemma 2.10, we have $I-\lambda_{n} B$ and $I-\mu_{n} C$ are nonexpansive. Thus, we obtain

$$
\begin{aligned}
\left\|y_{n}-p\right\| & =\left\|P_{E}\left(x_{n}-\lambda_{n} B x_{n}\right)-P_{E}\left(p-\lambda_{n} B p\right)\right\| \\
& \leq\left\|\left(x_{n}-\lambda_{n} B x_{n}\right)-\left(p-\lambda_{n} B p\right)\right\| \\
& =\left\|\left(I-\lambda_{n} B\right) x_{n}-\left(I-\lambda_{n} B\right) p\right\| \\
& \leq\left\|x_{n}-p\right\|, \\
\left\|z_{n}-p\right\| & =\left\|P_{E}\left(x_{n}-\mu_{n} C x_{n}\right)-P_{E}\left(p-\mu_{n} C p\right)\right\| \\
& \leq\left\|\left(x_{n}-\mu_{n} C x_{n}\right)-\left(p-\mu_{n} C p\right)\right\| \\
& =\left\|\left(I-\mu_{n} C\right) x_{n}-\left(I-\mu_{n} C\right) p\right\| \\
& \leq\left\|x_{n}-p\right\| .
\end{aligned}
$$

From Lemma 2.3, we have $S_{k}$ is nonexpansive with $F\left(S_{k}\right)=F(S)$. It follows that

$$
\begin{aligned}
\left\|t_{n}-p\right\| & =\left\|\alpha_{n}^{(1)} S_{k} x_{n}+\alpha_{n}^{(2)} y_{n}+\alpha_{n}^{(3)} z_{n}+\alpha_{n}^{(4)} u_{n}+\alpha_{n}^{(5)} v_{n}-p\right\| \\
& \leq \alpha_{n}^{(1)}\left\|S_{k} x_{n}-p\right\|+\alpha_{n}^{(2)}\left\|y_{n}-p\right\|+\alpha_{n}^{(3)}\left\|z_{n}-p\right\|+\alpha_{n}^{(4)}\left\|u_{n}-p\right\|+\alpha_{n}^{(5)}\left\|v_{n}-p\right\| \\
& \leq \alpha_{n}^{(1)}\left\|x_{n}-p\right\|+\alpha_{n}^{(2)}\left\|x_{n}-p\right\|+\alpha_{n}^{(3)}\left\|x_{n}-p\right\|+\alpha_{n}^{(4)}\left\|x_{n}-p\right\|+\alpha_{n}^{(5)}\left\|x_{n}-p\right\| \\
& =\left\|x_{n}-p\right\| .
\end{aligned}
$$

It follows that $p \in E_{k+1}$. This implies that $\Theta \subset E_{n}$ for each $n \geq 1$.

Step 3. We claim that $\lim _{n \rightarrow \infty}\left\|x_{n+1}-x_{n}\right\|=0$ and $\lim _{n \rightarrow \infty}\left\|x_{n}-t_{n}\right\|=0$.

From $x_{n}=P_{E_{n}} x_{0}$, we get

$$
\left\langle x_{0}-x_{n}, x_{n}-y\right\rangle \geq 0
$$

for each $y \in E_{n}$. Using $\Theta \subset E_{n}$, we have

$$
\left\langle x_{0}-x_{n}, x_{n}-p\right\rangle \geq 0 \text { for each } p \in \Theta, n \in \mathbb{N} \text {. }
$$


Hence, for $p \in \Theta$, we obtain

$$
\begin{aligned}
0 & \leq\left\langle x_{0}-x_{n}, x_{n}-p\right\rangle \\
& =\left\langle x_{0}-x_{n}, x_{n}-x_{0}+x_{0}-p\right\rangle \\
& =-\left\langle x_{0}-x_{n}, x_{0}-x_{n}\right\rangle+\left\langle x_{0}-x_{n}, x_{0}-p\right\rangle \\
& \leq-\left\|x_{0}-x_{n}\right\|^{2}+\left\|x_{0}-x_{n}\right\|\left\|x_{0}-p\right\| .
\end{aligned}
$$

It follows that

$$
\left\|x_{0}-x_{n}\right\| \leq\left\|x_{0}-p\right\|, \quad \forall p \in \Theta, n \in \mathbb{N} .
$$

From $x_{n}=P_{E_{n}} x_{0}$ and $x_{n+1}=P_{E_{n+1}} x_{0} \in E_{n+1} \subset E_{n}$, we have

$$
\left\langle x_{0}-x_{n}, x_{n}-x_{n+1}\right\rangle \geq 0 .
$$

For $n \in \mathbb{N}$, we compute

$$
\begin{aligned}
0 & \leq\left\langle x_{0}-x_{n}, x_{n}-x_{n+1}\right\rangle \\
& =\left\langle x_{0}-x_{n}, x_{n}-x_{0}+x_{0}-x_{n+1}\right\rangle \\
& =-\left\langle x_{0}-x_{n}, x_{0}-x_{n}\right\rangle+\left\langle x_{0}-x_{n}, x_{0}-x_{n+1}\right\rangle \\
& \leq-\left\|x_{0}-x_{n}\right\|^{2}+\left\langle x_{0}-x_{n}, x_{0}-x_{n+1}\right\rangle \\
& \leq-\left\|x_{0}-x_{n}\right\|^{2}+\left\|x_{0}-x_{n}\right\|\left\|x_{0}-x_{n+1}\right\|,
\end{aligned}
$$

and then

$$
\left\|x_{0}-x_{n}\right\| \leq\left\|x_{0}-x_{n+1}\right\|, \quad \forall n \in \mathbb{N} .
$$

Thus, the sequence $\left\{\left\|x_{n}-x_{0}\right\|\right\}$ is a bounded and nondecreasing sequence, so $\lim _{n \rightarrow \infty}\left\|x_{n}-x_{0}\right\|$ exists; that is, there exists $m$ such that

$$
m=\lim _{n \rightarrow \infty}\left\|x_{n}-x_{0}\right\|
$$


From (3.13), we get

$$
\begin{aligned}
\left\|x_{n}-x_{n+1}\right\|^{2} & =\left\|x_{n}-x_{0}+x_{0}-x_{n+1}\right\|^{2} \\
& =\left\|x_{n}-x_{0}\right\|^{2}+2\left\langle x_{n}-x_{0}, x_{0}-x_{n+1}\right\rangle+\left\|x_{0}-x_{n+1}\right\|^{2} \\
& =\left\|x_{n}-x_{0}\right\|^{2}+2\left\langle x_{n}-x_{0}, x_{0}-x_{n}+x_{n}-x_{n+1}\right\rangle+\left\|x_{0}-x_{n+1}\right\|^{2} \\
& =\left\|x_{n}-x_{0}\right\|^{2}+2\left\langle x_{n}-x_{0}, x_{0}-x_{n}\right\rangle+2\left\langle x_{n}-x_{0}, x_{n}-x_{n+1}\right\rangle+\left\|x_{0}-x_{n+1}\right\|^{2} \\
& =-\left\|x_{n}-x_{0}\right\|^{2}+2\left\langle x_{n}-x_{0}, x_{n}-x_{n+1}\right\rangle+\left\|x_{0}-x_{n+1}\right\|^{2} \\
& \leq-\left\|x_{n}-x_{0}\right\|^{2}+\left\|x_{0}-x_{n+1}\right\|^{2} .
\end{aligned}
$$

By (3.16), we obtain

$$
\lim _{n \rightarrow \infty}\left\|x_{n}-x_{n+1}\right\|=0
$$

Since $x_{n+1}=P_{E_{n+1}} x_{0} \in E_{n+1} \subset E_{n}$, we have

$$
\left\|x_{n}-t_{n}\right\| \leq\left\|x_{n}-x_{n+1}\right\|+\left\|x_{n+1}-t_{n}\right\| \leq 2\left\|x_{n}-x_{n+1}\right\| .
$$

By (3.18), we obtain

$$
\lim _{n \rightarrow \infty}\left\|x_{n}-t_{n}\right\|=0
$$

Step 4. We claim that the following statements hold:

(S1) $\lim _{n \rightarrow \infty}\left\|x_{n}-u_{n}\right\|=0$,

(S2) $\lim _{n \rightarrow \infty}\left\|x_{n}-y_{n}\right\|=0$,

(S3) $\lim _{n \rightarrow \infty}\left\|x_{n}-z_{n}\right\|=0$,

(S4) $\lim _{n \rightarrow \infty}\left\|x_{n}-v_{n}\right\|=0$.

For $p \in \Theta$, we note that

$$
\begin{aligned}
\left\|z_{n}-p\right\|^{2} & =\left\|P_{E}\left(x_{n}-\mu_{n} C x_{n}\right)-P_{E}\left(p-\mu_{n} C p\right)\right\|^{2} \\
& \leq\left\|\left(x_{n}-\mu_{n} C x_{n}\right)-\left(p-\mu_{n} C p\right)\right\|^{2} \\
& =\left\|\left(x_{n}-p\right)-\mu_{n}\left(C x_{n}-C p\right)\right\|^{2} \\
& \leq\left\|x_{n}-p\right\|^{2}-2 \mu_{n}\left\langle x_{n}-p, C x_{n}-C p\right\rangle+\mu_{n}^{2}\left\|C x_{n}-C p\right\|^{2} \\
& \leq\left\|x_{n}-p\right\|^{2}+\mu_{n}\left(\mu_{n}-2 \xi\right)\left\|C x_{n}-C p\right\|^{2} \\
& =\left\|x_{n}-p\right\|^{2}-\mu_{n}\left(2 \xi-\mu_{n}\right)\left\|C x_{n}-C p\right\|^{2} .
\end{aligned}
$$


Similarly, we also have

$$
\left\|y_{n}-p\right\|^{2} \leq\left\|x_{n}-p\right\|^{2}-\lambda_{n}\left(2 \beta-\lambda_{n}\right)\left\|B x_{n}-B \mathrm{p}\right\|^{2} .
$$

We note that

$$
\begin{aligned}
\left\|u_{n}-p\right\|^{2} & =\left\|T_{r_{n}}^{F_{1}}\left(I-r_{n} A_{1}\right) x_{n}-T_{r_{n}}^{F_{1}}\left(I-r_{n} A_{1}\right) p\right\|^{2} \\
& \leq\left\|\left(I-r_{n} A_{1}\right) x_{n}-\left(I-r_{n} A_{1}\right) p\right\|^{2} \\
& =\left\|\left(x_{n}-p\right)-r_{n}\left(A_{1} x_{n}-A_{1} p\right)\right\|^{2} \\
& =\left\|x_{n}-p\right\|^{2}-2 r_{n}\left\langle x_{n}-p, A_{1} x_{n}-A_{1} p\right\rangle+r_{n}^{2}\left\|A_{1} x_{n}-A_{1} p\right\|^{2} \\
& \leq\left\|x_{n}-p\right\|^{2}-2 r_{n} \rho\left\|A_{1} x_{n}-A_{1} p\right\|^{2}+r_{n}^{2}\left\|A_{1} x_{n}-A_{1} p\right\|^{2} \\
& =\left\|x_{n}-p\right\|^{2}+r_{n}\left(r_{n}-2 \rho\right)\left\|A_{1} x_{n}-A_{1} p\right\|^{2} \\
& =\left\|x_{n}-p\right\|^{2}-r_{n}\left(2 \rho-r_{n}\right)\left\|A_{1} x_{n}-A_{1} p\right\|^{2} .
\end{aligned}
$$

Similarly, we also have

$$
\left\|v_{n}-p\right\|^{2} \leq\left\|x_{n}-p\right\|^{2}-s_{n}\left(2 \omega-s_{n}\right)\left\|A_{2} x_{n}-A_{2} p\right\|^{2} .
$$

Observing that

$$
\begin{aligned}
\left\|t_{n}-p\right\|^{2} & \leq \alpha_{n}^{(1)}\left\|S_{k} x_{n}-p\right\|^{2}+\alpha_{n}^{(2)}\left\|y_{n}-p\right\|^{2}+\alpha_{n}^{(3)}\left\|z_{n}-p\right\|^{2}+\alpha_{n}^{(4)}\left\|u_{n}-p\right\|^{2}+\alpha_{n}^{(5)}\left\|v_{n}-p\right\|^{2} \\
& \leq \alpha_{n}^{(1)}\left\|x_{n}-p\right\|^{2}+\alpha_{n}^{(2)}\left\|y_{n}-p\right\|^{2}+\alpha_{n}^{(3)}\left\|z_{n}-p\right\|^{2}+\alpha_{n}^{(4)}\left\|u_{n}-p\right\|^{2}+\alpha_{n}^{(5)}\left\|v_{n}-p\right\|^{2}
\end{aligned}
$$

Substituting (3.21), (3.22), (3.23), and (3.24) into (3.25), we obtain

$$
\begin{aligned}
\left\|t_{n}-p\right\|^{2} \leq & \alpha_{n}^{(1)}\left\|x_{n}-p\right\|^{2}+\alpha_{n}^{(2)}\left\{\left\|x_{n}-p\right\|^{2}-\lambda_{n}\left(2 \beta-\lambda_{n}\right)\left\|B x_{n}-B p\right\|^{2}\right\} \\
& +\alpha_{n}^{(3)}\left\{\left\|x_{n}-p\right\|^{2}-\mu_{n}\left(2 \xi-\mu_{n}\right)\left\|C x_{n}-C p\right\|^{2}\right\} \\
& +\alpha_{n}^{(4)}\left\{\left\|x_{n}-p\right\|^{2}-r_{n}\left(2 \rho-r_{n}\right)\left\|A_{1} x_{n}-A_{1} p\right\|^{2}\right\} \\
& +\alpha_{n}^{(5)}\left\{\left\|x_{n}-p\right\|^{2}-s_{n}\left(2 \omega-s_{n}\right)\left\|A_{2} x_{n}-A_{2} p\right\|^{2}\right\} \\
= & \left\|x_{n}-p\right\|^{2}-\alpha_{n}^{(2)} \lambda_{n}\left(2 \beta-\lambda_{n}\right)\left\|B x_{n}-B p\right\|^{2}-\alpha_{n}^{(3)} \mu_{n}\left(2 \xi-\mu_{n}\right)\left\|C x_{n}-C p\right\|^{2} \\
& -\alpha_{n}^{(4)} r_{n}\left(2 \rho-r_{n}\right)\left\|A_{1} x_{n}-A_{1} p\right\|^{2}-\alpha_{n}^{(5)} s_{n}\left(2 \omega-s_{n}\right)\left\|A_{2} x_{n}-A_{2} p\right\|^{2} .
\end{aligned}
$$


It follows that

$$
\begin{aligned}
\alpha_{n}^{(3)} & \mu_{n}\left(2 \xi-\mu_{n}\right)\left\|C x_{n}-C p\right\|^{2} \\
& \leq\left\|x_{n}-p\right\|^{2}-\left\|t_{n}-p\right\|^{2}-\alpha_{n}^{(2)} \lambda_{n}\left(2 \beta-\lambda_{n}\right)\left\|B x_{n}-B p\right\|^{2} \\
& \quad-\alpha_{n}^{(4)} r_{n}\left(2 \rho-r_{n}\right)\left\|A_{1} x_{n}-A_{1} p\right\|^{2}-\alpha_{n}^{(5)} s_{n}\left(2 \omega-s_{n}\right)\left\|A_{2} x_{n}-A_{2} p\right\|^{2} \\
& \leq\left(\left\|x_{n}-p\right\|+\left\|t_{n}-p\right\|\right)\left\|x_{n}-t_{n}\right\| .
\end{aligned}
$$

From (C2), (C4), and (3.20), we have

$$
\lim _{n \rightarrow \infty}\left\|C x_{n}-C p\right\|=0 .
$$

Since $s_{n} \in(0,2 \omega)$, we also have

$$
\begin{aligned}
& \alpha_{n}^{(5)} s_{n}\left(2 \omega-s_{n}\right)\left\|A_{2} x_{n}-A_{2} p\right\|^{2} \\
& \quad \leq\left\|x_{n}-p\right\|^{2}-\left\|t_{n}-p\right\|^{2}-\alpha_{n}^{(2)} \lambda_{n}\left(2 \beta-\lambda_{n}\right)\left\|B x_{n}-B p\right\|^{2} \\
& \quad-\alpha_{n}^{(3)} \mu_{n}\left(2 \xi-\mu_{n}\right)\left\|C x_{n}-C p\right\|^{2}-\alpha_{n}^{(4)} r_{n}\left(2 \rho-r_{n}\right)\left\|A_{1} x_{n}-A_{1} p\right\|^{2} \\
& \quad \leq\left(\left\|x_{n}-p\right\|+\left\|t_{n}-p\right\|\right)\left\|x_{n}-t_{n}\right\| .
\end{aligned}
$$

From (C2), (C3), and (3.20), we obtain

$$
\lim _{n \rightarrow \infty}\left\|A_{2} x_{n}-A_{2} p\right\|=0
$$

Similarly, by (3.28) and (3.30), we can prove that

$$
\lim _{n \rightarrow \infty}\left\|B x_{n}-B p\right\|=\lim _{n \rightarrow \infty}\left\|A_{1} x_{n}-A_{1} p\right\|=0
$$


On the other hand, letting $p \in \Theta$ for each $n \geq 1$, we get $p=T_{r_{n}}^{F_{1}}\left(I-r_{n} A_{1}\right) p$. Since $T_{r_{n}}^{F_{1}}$ is firmly nonexpansive, we have

$$
\begin{aligned}
\left\|u_{n}-p\right\|^{2}= & \left\|T_{r_{n}}^{F_{1}}\left(I-r_{n} A_{1}\right) x_{n}-T_{r_{n}}^{F_{1}}\left(I-r_{n} A_{1}\right) p\right\|^{2} \\
\leq & \left\langle\left(I-r_{n} A_{1}\right) x_{n}-\left(I-r_{n} A_{1}\right) p, u_{n}-p\right\rangle \\
= & \frac{1}{2}\left\{\left\|\left(I-r_{n} A_{1}\right) x_{n}-\left(I-r_{n} A_{1}\right) p\right\|^{2}+\left\|u_{n}-p\right\|^{2}\right. \\
& \left.\quad-\left\|\left(I-r_{n} A_{1}\right) x_{n}-\left(I-r_{n} A_{1}\right) p-\left(u_{n}-p\right)\right\|^{2}\right\} \\
\leq & \frac{1}{2}\left\{\left\|x_{n}-p\right\|^{2}+\left\|u_{n}-p\right\|^{2}-\left\|\left(x_{n}-u_{n}\right)-r_{n}\left(A_{1} x_{n}-A_{1} p\right)\right\|^{2}\right\} \\
\leq & \frac{1}{2}\left\{\left\|x_{n}-p\right\|^{2}+\left\|u_{n}-p\right\|^{2}-\left\|x_{n}-u_{n}\right\|^{2}\right. \\
\quad & \left.+2 r_{n}\left\|x_{n}-u_{n}\right\|\left\|A_{1} x_{n}-A_{1} p\right\|-r_{n}^{2}\left\|A_{1} x_{n}-A_{1} p\right\|^{2}\right\} .
\end{aligned}
$$

So, we obtain

$$
\left\|u_{n}-p\right\|^{2} \leq\left\|x_{n}-p\right\|^{2}-\left\|x_{n}-u_{n}\right\|^{2}+2 r_{n}\left\|x_{n}-u_{n}\right\|\left\|A_{1} x_{n}-A_{1} p\right\|
$$

Observe that

$$
\begin{aligned}
\left\|y_{n}-p\right\|^{2}= & \left\|P_{E}\left(x_{n}-\lambda_{n} B x_{n}\right)-P_{E}\left(p-\lambda_{n} B p\right)\right\|^{2} \\
\leq & \left\langle\left(I-\lambda_{n} B\right) x_{n}-\left(I-\lambda_{n} B\right) p, y_{n}-p\right\rangle \\
= & \frac{1}{2}\left\{\left\|\left(I-\lambda_{n} B\right) x_{n}-\left(I-\lambda_{n} B\right) p\right\|^{2}+\left\|y_{n}-p\right\|^{2}\right. \\
& \left.\quad-\left\|\left(I-\lambda_{n} B\right) x_{n}-\left(I-\lambda_{n} B\right) p-\left(y_{n}-p\right)\right\|^{2}\right\} \\
\leq & \frac{1}{2}\left\{\left\|x_{n}-p\right\|^{2}+\left\|y_{n}-p\right\|^{2}-\left\|\left(x_{n}-y_{n}\right)-\lambda_{n}\left(B x_{n}-B p\right)\right\|^{2}\right\} \\
\leq & \frac{1}{2}\left\{\left\|x_{n}-p\right\|^{2}+\left\|y_{n}-p\right\|^{2}-\left\|x_{n}-y_{n}\right\|^{2}-\lambda_{n}^{2}\left\|B x_{n}-B p\right\|^{2}\right. \\
& \left.\quad+2 \lambda_{n}\left\langle x_{n}-y_{n}, B x_{n}-B p\right\rangle\right\},
\end{aligned}
$$

and hence

$$
\left\|y_{n}-p\right\|^{2} \leq\left\|x_{n}-p\right\|^{2}-\left\|x_{n}-y_{n}\right\|^{2}+2 \lambda_{n}\left\|x_{n}-y_{n}\right\|\left\|B x_{n}-B p\right\| .
$$


By using the same argument in (3.33) and (3.35), we can get

$$
\begin{gathered}
\left\|v_{n}-p\right\|^{2} \leq\left\|x_{n}-p\right\|^{2}-\left\|x_{n}-v_{n}\right\|^{2}+2 s_{n}\left\|x_{n}-v_{n}\right\|\left\|A_{2} x_{n}-A_{2} p\right\| \\
\left\|z_{n}-p\right\|^{2} \leq\left\|x_{n}-p\right\|^{2}-\left\|x_{n}-z_{n}\right\|^{2}+2 \mu_{n}\left\|x_{n}-z_{n}\right\|\left\|C x_{n}-C p\right\| .
\end{gathered}
$$

Substituting (3.33), (3.35), and (3.36) into (3.25), we obtain

$$
\begin{aligned}
\left\|t_{n}-p\right\|^{2} \leq & \alpha_{n}^{(1)}\left\|x_{n}-p\right\|^{2}+\alpha_{n}^{(2)}\left\|y_{n}-p\right\|^{2}+\alpha_{n}^{(3)}\left\|z_{n}-p\right\|^{2} \\
& +\alpha_{n}^{(4)}\left\|u_{n}-p\right\|^{2}+\alpha_{n}^{(5)}\left\|v_{n}-p\right\|^{2} \\
\leq & \alpha_{n}^{(1)}\left\|x_{n}-p\right\|^{2}+\alpha_{n}^{(2)}\left\{\left\|x_{n}-p\right\|^{2}-\left\|x_{n}-y_{n}\right\|^{2}+2 \lambda_{n}\left\|x_{n}-y_{n}\right\|\left\|B x_{n}-B p\right\|\right\} \\
& +\alpha_{n}^{(3)}\left\{\left\|x_{n}-p\right\|^{2}-\left\|x_{n}-z_{n}\right\|^{2}+2 \mu_{n}\left\|x_{n}-z_{n}\right\|\left\|C x_{n}-C p\right\|\right\} \\
& +\alpha_{n}^{(4)}\left\{\left\|x_{n}-p\right\|^{2}-\left\|x_{n}-u_{n}\right\|^{2}+2 r_{n}\left\|x_{n}-u_{n}\right\|\left\|A_{1} x_{n}-A_{1} p\right\|\right\} \\
& +\alpha_{n}^{(5)}\left\{\left\|x_{n}-p\right\|^{2}-\left\|x_{n}-v_{n}\right\|^{2}+2 s_{n}\left\|x_{n}-v_{n}\right\|\left\|A_{2} x_{n}-A_{2} p\right\|\right\} \\
= & \left\|x_{n}-p\right\|^{2}-\alpha_{n}^{(2)}\left\|x_{n}-y_{n}\right\|^{2}+2 \lambda_{n} \alpha_{n}^{(2)}\left\|x_{n}-y_{n}\right\|\left\|B x_{n}-B p\right\| \\
& -\alpha_{n}^{(3)}\left\|x_{n}-z_{n}\right\|^{2}+2 \mu_{n} \alpha_{n}^{(3)}\left\|x_{n}-z_{n}\right\|\left\|C x_{n}-C p\right\| \\
& -\alpha_{n}^{(4)}\left\|x_{n}-u_{n}\right\|^{2}+2 r_{n} \alpha_{n}^{(4)}\left\|x_{n}-u_{n}\right\|\left\|A_{1} x_{n}-A_{1} p\right\| \\
& -\alpha_{n}^{(5)}\left\|x_{n}-v_{n}\right\|^{2}+2 s_{n} \alpha_{n}^{(5)}\left\|x_{n}-v_{n}\right\|\left\|A_{2} x_{n}-A_{2} p\right\| .
\end{aligned}
$$

It follows that

$$
\begin{aligned}
\alpha_{n}^{(4)}\left\|x_{n}-u_{n}\right\|^{2} \leq & \left\|x_{n}-p\right\|^{2}-\left\|t_{n}-p\right\|^{2}-\alpha_{n}^{(2)}\left\|x_{n}-y_{n}\right\|^{2}+2 \lambda_{n} \alpha_{n}^{(2)}\left\|x_{n}-y_{n}\right\|\left\|B x_{n}-B p\right\| \\
& -\alpha_{n}^{(3)}\left\|x_{n}-z_{n}\right\|^{2}+2 \mu_{n} \alpha_{n}^{(3)}\left\|x_{n}-z_{n}\right\|\left\|C x_{n}-C p\right\| \\
& +2 r_{n} \alpha_{n}^{(4)}\left\|x_{n}-u_{n}\right\|\left\|A_{1} x_{n}-A_{1} p\right\|-\alpha_{n}^{(5)}\left\|x_{n}-v_{n}\right\|^{2} \\
& +2 s_{n} \alpha_{n}^{(5)}\left\|x_{n}-v_{n}\right\|\left\|A_{2} x_{n}-A_{2} p\right\| \\
\leq & \left(\left\|x_{n}-p\right\|+\left\|t_{n}-p\right\|\right)\left\|x_{n}-t_{n}\right\|+2 \lambda_{n} \alpha_{n}^{(2)}\left\|x_{n}-y_{n}\right\|\left\|B x_{n}-B p\right\| \\
& +2 \mu_{n} \alpha_{n}^{(3)}\left\|x_{n}-z_{n}\right\|\left\|C x_{n}-C p\right\|+2 r_{n} \alpha_{n}^{(4)}\left\|x_{n}-u_{n}\right\|\left\|A_{1} x_{n}-A_{1} p\right\| \\
& +2 s_{n} \alpha_{n}^{(5)}\left\|x_{n}-v_{n}\right\|\left\|A_{2} x_{n}-A_{2} p\right\| .
\end{aligned}
$$


From (C2), (3.20), (3.28), (3.30), and (3.31), we have

$$
\lim _{n \rightarrow \infty}\left\|x_{n}-u_{n}\right\|=0
$$

By using the same argument, we can prove that

$$
\lim _{n \rightarrow \infty}\left\|x_{n}-y_{n}\right\|=\lim _{n \rightarrow \infty}\left\|x_{n}-z_{n}\right\|=\lim _{n \rightarrow \infty}\left\|x_{n}-v_{n}\right\|=0 .
$$

Applying (3.20), (3.39), and (3.40), we can obtain

$$
\lim _{n \rightarrow \infty}\left\|t_{n}-u_{n}\right\|=\lim _{n \rightarrow \infty}\left\|t_{n}-y_{n}\right\|=\lim _{n \rightarrow \infty}\left\|t_{n}-z_{n}\right\|=\lim _{n \rightarrow \infty}\left\|t_{n}-v_{n}\right\|=0 .
$$

Step 5. We show that

$$
z \in F(S) \cap \operatorname{GMEP}\left(F_{1}, \varphi, A_{1}\right) \cap \operatorname{GMEP}\left(F_{2}, \varphi, A_{2}\right) \cap \operatorname{VI}(E, B) \cap \operatorname{VI}(E, C) .
$$

Assume that $\lambda_{n} \rightarrow \lambda \in[c, 2 \beta]$ and $\mu_{n} \rightarrow \mu \in[d, 2 \xi]$.

Define a mapping $D: E \rightarrow E$ by

$$
\begin{aligned}
D x= & \alpha^{(1)} S_{k} x+\alpha^{(2)} P_{E}(1-\lambda B) x+\alpha^{(3)} P_{E}(1-\mu C) x+\alpha^{(4)} T_{r}^{F_{1}}\left(I-r A_{1}\right) x \\
& +\alpha^{(5)} T_{s}^{F_{2}}\left(I-s A_{2}\right) x, \quad \forall x \in E,
\end{aligned}
$$

where $\lim _{n \rightarrow \infty} \alpha_{n}^{(i)}=\alpha^{(i)} \in(0,1)$, when $i=1,2,3,4,5$. By (C1), then we have $\sum_{i=1}^{5} \alpha_{n}^{(i)}=1$. From Lemma 2.8, we have $D$ is nonexpansive and

$$
\begin{aligned}
F(D) & =F\left(S_{k}\right) \cap F\left(P_{E}(1-\lambda B)\right) \cap F\left(P_{E}(1-\mu C)\right) \cap F\left(T_{r}^{F_{1}}\left(I-r A_{1}\right)\right) \cap F\left(T_{s}^{F_{2}}\left(I-s A_{2}\right)\right) \\
& =F\left(S_{k}\right) \cap \operatorname{GMEP}\left(F_{1}, \varphi, A_{1}\right) \cap \operatorname{GMEP}\left(F_{2}, \varphi, A_{2}\right) \cap \operatorname{VI}(E, B) \cap \operatorname{VI}(E, C) .
\end{aligned}
$$


We note that

$$
\begin{aligned}
\left\|P x_{n}-x_{n}\right\| \leq & \left\|P x_{n}-t_{n}\right\|+\left\|t_{n}-x_{n}\right\| \\
= & \|\left[\alpha^{(1)} S_{k} x_{n}+\alpha^{(2)} P_{E}(1-\lambda B) x_{n}+\alpha^{(3)} P_{E}(1-\mu C) x_{n}\right. \\
& \left.+\alpha^{(4)} T_{r}^{F_{1}}\left(I-r A_{1}\right) x_{n}+\alpha^{(5)} T_{s}^{F_{2}}\left(I-s A_{2}\right) x_{n}\right] \\
& \quad-\left[\alpha_{n}^{(1)} S_{k} x_{n}+\alpha_{n}^{(2)} P_{E}\left(1-\lambda_{n} B\right) x_{n}+\alpha_{n}^{(3)} P_{E}\left(1-\mu_{n} C\right) x_{n}\right. \\
& \left.+\alpha_{n}^{(4)} T_{r}^{F_{1}}\left(I-r A_{1}\right) x_{n}+\alpha_{n}^{(5)} T_{s}^{F_{2}}\left(I-s A_{2}\right) x_{n}\right]\|+\| t_{n}-x_{n} \| \\
\leq & \left|\alpha^{(1)}-\alpha_{n}^{(1)}\right|\left\|S_{k} x_{n}\right\|+\alpha^{(2)}\left\|P_{E}(I-\lambda B) x_{n}-P_{E}\left(I-\lambda_{n} B\right) x_{n}\right\| \\
& +\left|\alpha^{(2)}-\alpha_{n}^{(2)}\right|\left\|P_{E}\left(I-\lambda_{n} B\right) x_{n}\right\| \\
& +\alpha^{(3)}\left\|P_{E}(I-\mu C) x_{n}-P_{E}\left(I-\mu_{n} C\right) x_{n}\right\|+\left|\alpha^{(3)}-\alpha_{n}^{(3)}\right|\left\|P_{E}\left(I-\mu_{n} C\right) x_{n}\right\| \\
& +\left|\alpha^{(4)}-\alpha_{n}^{(4)}\right|\left\|T_{r}^{F_{1}}\left(I-r A_{1}\right) x_{n}\right\|+\left|\alpha^{(5)}-\alpha_{n}^{(5)}\right|\left\|T_{s}^{F_{2}}\left(I-s A_{2}\right) x_{n}\right\|+\left\|t_{n}-x_{n}\right\| \\
\leq & \left|\alpha^{(1)}-\alpha_{n}^{(1)}\right|\left\|S_{k} x_{n}\right\|+\alpha^{(2)}\left|\lambda_{n}-\lambda\right|\left\|B x_{n}\right\|+\left|\alpha^{(2)}-\alpha_{n}^{(2)}\right|\left\|P_{E}\left(I-\lambda_{n} B\right) x_{n}\right\| \\
& +\alpha^{(3)}\left|\mu_{n}-\mu\right|\left\|C x_{n}\right\|+\left|\alpha^{(3)}-\alpha_{n}^{(3)}\right|\left\|P_{E}\left(I-\mu_{n} C\right) x_{n}\right\| \\
& +\left|\alpha^{(4)}-\alpha_{n}^{(4)}\right|\left\|T_{r}^{F_{1}}\left(I-r A_{1}\right) x_{n}\right\|+\left|\alpha^{(5)}-\alpha_{n}^{(5)}\right|\left\|T_{s}^{F_{2}}\left(I-s A_{2}\right) x_{n}\right\|+\left\|t_{n}-x_{n}\right\| \\
\leq & K_{1}\left(\sum_{i=1}^{5}\left|\alpha^{(i)}-\alpha_{n}^{(i)}\right|+\left|\lambda_{n}-\lambda\right|+\left|\mu_{n}-\mu\right|\right)+\left\|t_{n}-x_{n}\right\|,
\end{aligned}
$$

where $K_{1}$ is an appropriate constant such that

$$
\begin{gathered}
K_{1}=\max \left\{\sup _{n \geq 1}\left\|T_{r}^{F_{1}}\left(I-r A_{1}\right) x_{n}\right\|, \sup _{n \geq 1}\left\|T_{s}^{F_{2}}\left(I-s A_{2}\right) x_{n}\right\|, \sup _{n \geq 1}\left\|P_{E}\left(I-\lambda_{n} B\right) x_{n}\right\|,\right. \\
\left.\sup _{n \geq 1}\left\|P_{E}\left(I-\mu_{n} C\right) x_{n}\right\|, \sup _{n \geq 1}\left\|B x_{n}\right\|, \sup _{n \geq 1}\left\|C x_{n}\right\|, \sup _{n \geq 1}\left\|S_{k} x_{n}\right\|\right\} .
\end{gathered}
$$

From (C2), (C5), and (3.20), we obtain

$$
\lim _{n \rightarrow \infty}\left\|x_{n}-D x_{n}\right\|=0
$$


Since $\left\{x_{n_{i}}\right\}$ is bounded, there exists a subsequence $\left\{x_{n_{i}}\right\}$ of $\left\{x_{n}\right\}$ which converges weakly to $z$. Without loss of generality, we may assume that $\left\{x_{n_{i}}\right\} \rightarrow z$. It follows from (3.47), that

$$
\lim _{n \rightarrow \infty}\left\|x_{n_{i}}-p x_{n_{i}}\right\|=0
$$

It follows from Lemma 2.4 that $z \in F(D)$. By (3.44), we have $z \in \Theta$.

Step 6. Finally, we show that $x_{n} \rightarrow z$, where $z=P_{\Theta} x_{0}$.

Since $\Theta$ is nonempty closed convex subset of $H$, there exists a unique $z^{\prime} \in \Theta$ such that $z^{\prime}=P_{\Theta} x_{0}$. Since $z^{\prime} \in \Theta \subset E_{n}$ and $x_{n}=P_{E_{n}} x_{0}$, we have

$$
\left\|x_{0}-x_{n}\right\|=\left\|x_{0}-P_{E_{n}} x_{0}\right\| \leq\left\|x_{0}-z^{\prime}\right\|
$$

for all $n \geq 1$. From (3.49), $\left\{x_{n}\right\}$ is bounded, so $\omega_{w}\left(x_{n}\right) \neq \emptyset$. By the weak lower semicontinuity of the norm, we have

$$
\left\|x_{0}-z\right\| \leq \liminf _{i \rightarrow \infty}\left\|x_{0}-x_{n_{i}}\right\| \leq\left\|x_{0}-z^{\prime}\right\|
$$

Since $z \in \omega_{w}\left(x_{n}\right) \subset \Theta$, we obtain

$$
\left\|x_{0}-z^{\prime}\right\|=\left\|x_{0}-P_{\Theta} x_{0}\right\| \leq\left\|x_{0}-z\right\| .
$$

Using (3.49) and (3.50), we obtain $z^{\prime}=z$. Thus, $\omega_{w}\left(x_{n}\right)=\{z\}$ and $x_{n} \rightarrow z$. So we have

$$
\left\|x_{0}-z^{\prime}\right\| \leq\left\|x_{0}-z\right\| \leq \liminf _{i \rightarrow \infty}\left\|x_{0}-x_{n}\right\| \leq \limsup _{i \rightarrow \infty}\left\|x_{0}-x_{n}\right\| \leq\left\|x_{0}-z^{\prime}\right\| .
$$

Thus,

$$
\left\|x_{0}-z\right\|=\lim _{i \rightarrow \infty}\left\|x_{0}-x_{n}\right\|=\left\|x_{0}-z^{\prime}\right\|
$$

From $x_{n} \rightarrow z$, we obtain $\left(x_{0}-x_{n}\right) \rightarrow\left(x_{0}-z\right)$. Using Lemma 2.5, we obtain that

$$
\left\|x_{n}-z\right\|=\left\|\left(x_{n}-x_{0}\right)-\left(z-x_{0}\right)\right\| \longrightarrow 0
$$

as $n \rightarrow \infty$ and hence $x_{n} \rightarrow z$ in norm. This completes the proof.

If the mapping $S$ is nonexpansive, then $S_{k}=S_{0}=S$. We can obtain the following result from Theorem 3.1 immediately.

Corollary 3.2. Let $E$ be a nonempty closed convex subset of a real Hilbert space $H$. Let $F_{1}$ and $F_{2}$ be two bifunctions from $E \times E$ to $\mathbb{R}$ satisfying (A1)-(A5), and let $\varphi: E \rightarrow \mathbb{R} \cup\{+\infty\}$ be a proper lower semicontinuous and convex function with either (B1) or (B2). Let $A_{1}, A_{2}, B, C$ be four $\rho, \omega, \beta$, 
$\xi$-inverse-strongly monotone mappings of $E$ into $H$, respectively. Let $S: E \rightarrow E$ be a nonexpansive mapping with a fixed point. Suppose that

$$
\Theta:=F(S) \cap \operatorname{GMEP}\left(F_{1}, \varphi, A_{1}\right) \cap \operatorname{GMEP}\left(F_{2}, \varphi, A_{2}\right) \cap \operatorname{VI}(E, B) \cap \operatorname{VI}(E, C) \neq \emptyset .
$$

Let $\left\{x_{n}\right\}$ be a sequence generated by the following iterative algorithm (3.1), where $\left\{\alpha_{n}^{(i)}\right\}$ are sequences in $(0,1)$, where $i=1,2,3,4,5, r_{n} \in(0,2 \rho), s_{n} \in(0,2 \omega)$, and $\left\{\lambda_{n}\right\},\left\{\mu_{n}\right\}$ are positive sequences. Assume that the control sequences satisfy (C1)-(C5) in Theorem 3.1. Then, $\left\{x_{n}\right\}$ converges strongly to $P_{\Theta} x_{0}$.

If $\varphi=0$ and $A_{1}=A_{2}=0$ in Theorem 3.1, then we can obtain the following result immediately.

Corollary 3.3. Let $E$ be a nonempty closed convex subset of a real Hilbert space $H$. Let $F_{1}$ and $F_{2}$ be two bifunctions from $E \times E$ to $\mathbb{R}$ satisfying (A1)-(A5), and let $\varphi: E \rightarrow \mathbb{R} \cup\{+\infty\}$ be a proper lower semicontinuous and convex function with either (B1) or (B2). Let $B, C$ be two $\beta$, $\xi$-inverse-strongly monotone mappings of $E$ into $H$, respectively. Let $S: E \rightarrow E$ be a nonexpansive mapping with a fixed point. Suppose that

$$
\Theta:=F(S) \cap \operatorname{EP}\left(F_{1}\right) \cap \operatorname{EP}\left(F_{2}\right) \cap \operatorname{VI}(E, B) \cap \operatorname{VI}(E, C) \neq \emptyset .
$$

Let $\left\{x_{n}\right\}$ be a sequence generated by the following iterative algorithm:

$$
\begin{gathered}
x_{0} \in H, \quad E_{1}=E, \quad x_{1}=P_{E_{1}} x_{0}, \quad u_{n} \in E, \quad v_{n} \in E, \\
F_{1}\left(u_{n}, u\right)+\frac{1}{r_{n}}\left\langle u-u_{n}, u_{n}-x_{n}\right\rangle \geq 0, \quad \forall u \in E, \\
F_{2}\left(v_{n}, v\right)+\frac{1}{s_{n}}\left\langle v-v_{n}, v_{n}-x_{n}\right\rangle \geq 0, \quad \forall v \in E, \\
z_{n}=P_{E}\left(x_{n}-\mu_{n} C x_{n}\right), \\
y_{n}=P_{E}\left(x_{n}-\lambda_{n} B x_{n}\right), \\
t_{n}=\alpha_{n}^{(1)} S x_{n}+\alpha_{n}^{(2)} y_{n}+\alpha_{n}^{(3)} z_{n}+\alpha_{n}^{(4)} u_{n}+\alpha_{n}^{(5)} v_{n}, \\
E_{n+1}=\left\{w \in E_{n}:\left\|t_{n}-w\right\| \leq\left\|x_{n}-w\right\|\right\}, \\
x_{n+1}=P_{E_{n+1}} x_{0}, \quad \forall n \geq 1,
\end{gathered}
$$

where $\left\{\alpha_{n}^{(i)}\right\}$ are sequences in $(0,1)$, where $i=1,2,3,4,5, r_{n} \in(0, \infty), s_{n} \in(0, \infty)$ and $\left\{\lambda_{n}\right\},\left\{\mu_{n}\right\}$ are positive sequences. Assume that the control sequences satisfy the condition (C1)-(C5) in Theorem 3.1. Then, $\left\{x_{n}\right\}$ converges strongly to $P_{\Theta} x_{0}$.

If $B=0, C=0$, and $F_{1}\left(u_{n}, u\right)=F_{1}\left(v_{n}, v\right)=0$ in Corollary 3.3, then $P_{E}=I$ and we get $u_{n}=y_{n}=x_{n}$ and $v_{n}=z_{n}=x_{n}$; hence, we can obtain the following result immediately.

Corollary 3.4. Let E be a nonempty closed convex subset of a real Hilbert space $H$. Let $S: E \rightarrow E$ be a $k$-strictly pseudocontractive mapping with a fixed point. Define a mapping $S_{k}: E \rightarrow E$ by $S_{k} x=$ $k x+(1-k) S x$, for all $x \in E$. Suppose that $F(S) \neq \emptyset$. Let $\left\{x_{n}\right\}$ be a sequence generated by the following 
iterative algorithm:

$$
\begin{gathered}
x_{0} \in H, \quad E_{1}=E, \quad x_{1}=P_{E_{1}} x_{0}, \\
t_{n}=\alpha_{n} S_{k} x_{n}+\left(1-\alpha_{n}\right) x_{n}, \\
E_{n+1}=\left\{w \in E_{n}:\left\|t_{n}-w\right\| \leq\left\|x_{n}-w\right\|\right\}, \\
x_{n+1}=P_{E_{n+1}} x_{0}, \quad \forall n \geq 1,
\end{gathered}
$$

where $\left\{\alpha_{n}\right\}$ are sequences in $(0,1)$. Assume that the control sequences satisfy the condition $\lim _{n \rightarrow \infty} \alpha_{n}=\alpha \in(0,1)$ in Theorem 3.1. Then, $\left\{x_{n}\right\}$ converges strongly to a point $P_{F(S)} x_{0}$.

\section{Convex Feasibility Problem}

Finally, we consider the following Convex Feasibility Problem (CFP): finding an $x \in \bigcap_{j=1}^{M} C_{j}$, where $M \geq 1$ is an integer and each $C_{i}$ is assumed to be the solutions of equilibrium problem with the bifunction $F_{j}, j=1,2,3, \ldots, M$ and the solution set of the variational inequality problem. There is a considerable investigation on CFP in the setting of Hilbert spaces which captures applications in various disciplines such as image restoration $[35,36]$, computer tomography [37], and radiation therapy treatment planning [38].

The following result can be obtained from Theorem 3.1. We, therefore, omit the proof.

Theorem 4.1. Let $E$ be a nonempty closed convex subset of a real Hilbert space $H$. Let $\left\{F_{j}\right\}_{j=1}^{M}$ be a family of bifunction from $E \times E$ to $\mathbb{R}$ satisfying (A1)-(A5), and let $\varphi: E \rightarrow \mathbb{R} \cup\{+\infty\}$ be a proper lower semicontinuous and convex function with either (B1) or (B2). Let $A_{j}: E \rightarrow H$ be $\rho_{j}$-inversestrongly monotone mapping for each $j \in\{1,2,3, \ldots, M\}$. Let $B_{i}: E \rightarrow H$ be $\beta_{i}$-inverse-strongly monotone mapping for each $i \in\{1,2,3, \ldots, N\}$. Let $S: E \rightarrow E$ be a $k$-strictly pseudocontractive mapping with a fixed point. Define a mapping $S_{k}: E \rightarrow E$ by $S_{k} x=k x+(1-k) S x$, for all $x \in E$. Suppose that

$$
\Theta:=F(S) \cap\left(\bigcap_{j=1}^{M} \operatorname{GMEP}\left(F_{j}, \varphi, A_{j}\right)\right) \cap\left(\bigcap_{i=1}^{N} \operatorname{VI}\left(E, B_{i}\right)\right) \neq \emptyset
$$

Let $\left\{x_{n}\right\}$ be a sequence generated by the following iterative algorithm:

$$
\begin{gathered}
x_{0} \in H, \quad E_{1}=E, \quad x_{1}=P_{E_{1}} x_{0}, \quad v_{1}, v_{2}, \ldots, v_{M} \in E, \\
F_{1}\left(v_{n, 1}, v_{1}\right)+\varphi\left(v_{1}\right)-\varphi\left(v_{n, 1}\right)+\left\langle A_{1} x_{n}, v_{1}-v_{n, 1}\right\rangle+\frac{1}{r_{1}}\left\langle v_{1}-v_{n, 1}, v_{n, 1}-x_{n}\right\rangle \geq 0, \quad \forall v_{1} \in E,
\end{gathered}
$$




$$
\begin{gathered}
F_{2}\left(v_{n, 2}, v_{2}\right)+\varphi\left(v_{2}\right)-\varphi\left(v_{n, 2}\right)+\left\langle A_{2} x_{n}, v_{2}-v_{n, 2}\right\rangle+\frac{1}{r_{2}}\left\langle v_{2}-v_{n, 2}, v_{n, 2}-x_{n}\right\rangle \geq 0, \quad \forall v_{2} \in E, \\
\vdots \\
F_{M}\left(v_{n, M}, v_{M}\right)+\varphi\left(v_{M}\right)-\varphi\left(v_{n, M}\right)+\left\langle A_{M} x_{n}, v_{M}-v_{n, M}\right\rangle \\
+\frac{1}{r_{M}}\left\langle v_{M}-v_{n, M}, v_{n, M}-x_{n}\right\rangle \geq 0, \quad \forall v_{M} \in E, \\
y_{n, 1}=P_{E}\left(x_{n}-\lambda_{n, 1} B_{1} x_{n}\right), \\
y_{n, 2}=P_{E}\left(x_{n}-\lambda_{n, 2} B_{2} x_{n}\right), \\
\vdots \\
y_{n, N}=P_{E}\left(x_{n}-\lambda_{n, N} B_{N} x_{n}\right), \\
t_{n}=\alpha_{n, 0} S_{k} x_{n}+\sum_{i=1}^{N} \alpha_{n, i} y_{n, i}+\sum_{j=1}^{M} \alpha_{n, j}^{\prime} v_{n, j}, \\
E_{n+1}=\left\{w \in E_{n}:\left\|t_{n}-w\right\| \leq\left\|x_{n}-w\right\|\right\}, \\
x_{n+1}=P_{E_{n+1}} x_{0}, \quad \forall n \geq 1,
\end{gathered}
$$

where $\alpha_{n, 0}, \alpha_{n, 1}, \alpha_{n, 2}, \ldots, \alpha_{n, N}$ and $\alpha_{n, 1}^{\prime}, \alpha_{n, 2}^{\prime}, \ldots, \alpha_{n, M}^{\prime} \in(0,1)$ such that $\sum_{i=0}^{N} \alpha_{n, i}+\sum_{j=1}^{M} \alpha_{n, j}^{\prime}=1$, $\left\{\lambda_{n, i}\right\}$ are positive sequences in $(0,1)$. Assume that the control sequences satisfy the following restrictions:

(C1) $\lim _{n \rightarrow \infty} \alpha_{n}^{(i)}=\alpha^{(i)} \in(0,1)$, for each $0 \leq i \leq N$,

(C2) $\lim _{n \rightarrow \infty} \alpha_{n}^{\prime(j)}=\alpha^{\prime(j)} \in(0,1)$, for each $1 \leq j \leq M$,

(C3) $a_{j} \leq r_{j} \leq 2 \rho_{j}$, where $a_{j}$ is some positive constants for each $1 \leq j \leq M$,

(C4) $c_{i} \leq \lambda_{n, i} \leq 2 \beta_{i}$, where $c_{i}$ is some positive constants for each $1 \leq i \leq N$,

(C5) $\lim _{n \rightarrow \infty}\left|\lambda_{n+1, i}-\lambda_{n, i}\right|=0$, for each $1 \leq i \leq N$.

Then, $\left\{x_{n}\right\}$ converges strongly to $P_{\Theta} x_{0}$.

If $A_{j}=0$, for each $1 \leq j \leq M$ and $F_{i}\left(v_{n, i}, v_{i}\right)=0$, for each $1 \leq i \leq N$ in Theorem 4.1, then $v_{n, i}=x_{n}$; hence, we can obtain the following result immediately.

Theorem 4.2. Let $E$ be a nonempty closed convex subset of a real Hilbert space $H$. Let $\varphi: E \rightarrow \mathbb{R} \cup$ $\{+\infty\}$ be a proper lower semicontinuous and convex function with either (B1) or (B2). Let $B_{i}: E \rightarrow H$ be $\beta_{i}$-inverse-strongly monotone mapping for each $i \in\{1,2,3, \ldots, N\}$. Let $S: E \rightarrow E$ be a $k$-strictly pseudocontractive mapping with a fixed point. Define a mapping $S_{k}: E \rightarrow E$ by $S_{k} x=k x+(1-k) S x$, for all $x \in E$. Suppose that

$$
\Theta:=F(S) \cap\left(\bigcap_{i=1}^{N} \operatorname{VI}\left(E, B_{i}\right)\right) \neq \emptyset .
$$


Let $\left\{x_{n}\right\}$ be a sequence generated by the following iterative algorithm:

$$
\begin{gathered}
x_{0} \in H, \quad E_{1}=E, \quad x_{1}=P_{E_{1}} x_{0}, \\
y_{n, 1}=P_{E}\left(x_{n}-\lambda_{n, 1} B_{1} x_{n}\right), \\
y_{n, 2}=P_{E}\left(x_{n}-\lambda_{n, 2} B_{2} x_{n}\right), \\
\vdots \\
y_{n, N}=P_{E}\left(x_{n}-\lambda_{n, N} B_{N} x_{n}\right), \\
t_{n}=\alpha_{n, 0} S_{k} x_{n}+\sum_{i=1}^{N} \alpha_{n, i} y_{n, i}, \\
E_{n+1}=\left\{w \in E_{n}:\left\|t_{n}-w\right\| \leq\left\|x_{n}-w\right\|\right\}, \\
x_{n+1}=P_{E_{n+1}} x_{0}, \quad \forall n \geq 1,
\end{gathered}
$$

where $\alpha_{n, 0}, \alpha_{n, 1}, \alpha_{n, 2}, \ldots, \alpha_{n, N} \in(0,1)$ such that $\sum_{i=0}^{N} \alpha_{n, i}=1,\left\{\lambda_{n, i}\right\}$ are positive sequences in $(0,1)$. Assume that the control sequences satisfy the following restrictions:

(C1) $\lim _{n \rightarrow \infty} \alpha_{n}^{(i)}=\alpha^{(i)} \in(0,1)$, for each $0 \leq i \leq N$,

(C2) $c_{i} \leq \lambda_{n, i} \leq 2 \beta_{i}$, where $c_{i}$ is some positive constants for each $1 \leq i \leq N$,

(C3) $\lim _{n \rightarrow \infty}\left|\lambda_{n+1, i}-\lambda_{n, i}\right|=0$, for each $1 \leq i \leq N$.

Then, $\left\{x_{n}\right\}$ converges strongly to $P_{\Theta} x_{0}$.

If $B_{i}=0$, for each $1 \leq i \leq N$ in Theorem 4.1, then we get $y_{n, i}=x_{n}$. Hence, we can obtain the following result immediately.

Theorem 4.3. Let E be a nonempty closed convex subset of a real Hilbert space $H$. Let be a $\left\{F_{j}\right\}_{j=1}^{M}$ be a family of bifunction from $E \times E$ to $\mathbb{R}$ satisfying $(A 1)-(A 5)$, and let $\varphi: E \rightarrow \mathbb{R} \cup\{+\infty\}$ be a proper lower semicontinuous and convex function with either (B1) or (B2). Let $A_{j}: E \rightarrow H$ be $\rho_{j}$-inverse-strongly monotone mapping for each $j \in\{1,2,3, \ldots, M\}$. Let $S: E \rightarrow E$ be a $k$-strictly pseudocontractive mapping with a fixed point. Define a mapping $S_{k}: E \rightarrow E$ by $S_{k} x=k x+(1-k) S x$, for all $x \in E$. Suppose that

$$
\Theta:=F(S) \cap\left(\bigcap_{j=1}^{M} \operatorname{GMEP}\left(F_{j}, \varphi, A_{j}\right)\right) \neq \emptyset .
$$


Let $\left\{x_{n}\right\}$ be a sequence generated by the following iterative algorithm:

$$
\begin{gathered}
x_{0} \in H, \quad E_{1}=E, \quad x_{1}=P_{E_{1}} x_{0}, \quad v_{1}, v_{2}, \ldots, v_{M} \in E, \\
F_{1}\left(v_{n, 1}, v_{1}\right)+\varphi\left(v_{1}\right)-\varphi\left(v_{n, 1}\right)+\left\langle A_{1} x_{n}, v_{1}-v_{n, 1}\right\rangle+\frac{1}{r_{1}}\left\langle v_{1}-v_{n, 1}, v_{n, 1}-x_{n}\right\rangle \geq 0, \quad \forall v_{1} \in E, \\
F_{2}\left(v_{n, 2}, v_{2}\right)+\varphi\left(v_{2}\right)-\varphi\left(v_{n, 2}\right)+\left\langle A_{2} x_{n}, v_{2}-v_{n, 2}\right\rangle+\frac{1}{r_{2}}\left\langle v_{2}-v_{n, 2}, v_{n, 2}-x_{n}\right\rangle \geq 0, \quad \forall v_{2} \in E, \\
\vdots \\
F_{M}\left(v_{n, M}, v_{M}\right)+\varphi\left(v_{M}\right)-\varphi\left(v_{n, M}\right)+\left\langle A_{M} x_{n}, v_{M}-v_{n, M}\right\rangle \\
+\frac{1}{r_{M}}\left\langle v_{M}-v_{n, M}, v_{n, M}-x_{n}\right\rangle \geq 0, \quad \forall v_{M} \in E, \\
t_{n}=\alpha_{n, 0} S_{k} x_{n}+\sum_{j=1}^{M} \alpha_{n, j}^{\prime} v_{n, j}, \\
E_{n+1}=\left\{w \in E_{n}:\left\|t_{n}-w\right\| \leq\left\|x_{n}-w\right\|\right\}, \\
x_{n+1}=P_{E_{n+1}} x_{0}, \quad \forall n \geq 1,
\end{gathered}
$$

where $\alpha_{n, 0}$ and $\alpha_{n, 1}^{\prime}, \alpha_{n, 2}^{\prime}, \ldots, \alpha_{n, M}^{\prime} \in(0,1)$ such that $\alpha_{n, 0}+\sum_{j=1}^{M} \alpha_{n, j}^{\prime}=1$. Assume that the control sequences satisfy the following restrictions:

(C1) $\lim _{n \rightarrow \infty} \alpha_{n}^{(0)}=\alpha^{(0)} \in(0,1)$,

(C2) $\lim _{n \rightarrow \infty} \alpha_{n}^{\prime(j)}=\alpha^{\prime(j)} \in(0,1)$, for each $1 \leq j \leq M$,

(C3) $a_{j} \leq r_{j} \leq 2 \rho_{j}$, where $a_{j}$ is some positive constants for each $1 \leq j \leq M$.

Then, $\left\{x_{n}\right\}$ converges strongly to $P_{\Theta} x_{0}$.

\section{Acknowledgments}

The authors would like to thank the anonymous referees for helpful comments to improve this paper, and the second author was supported by the Commission on Higher Education and the Thailand Research Fund under Grant MRG5380044. Moreover, they also would like to thank the National Research University Project of Thailand's Office of the Higher Education Commission for financial support under NRU-CSEC Project no. 54000267.

\section{References}

[1] H. Zhou, "Convergence theorems of fixed points for $\kappa$-strict pseudo-contractions in Hilbert spaces," Nonlinear Analysis. Theory, Methods E Applications, vol. 69, no. 2, pp. 456-462, 2008.

[2] L.-C. Ceng and J.-C. Yao, "A hybrid iterative scheme for mixed equilibrium problems and fixed point problems," Journal of Computational and Applied Mathematics, vol. 214, no. 1, pp. 186-201, 2008.

[3] E. Blum and W. Oettli, "From optimization and variational inequalities to equilibrium problems," The Mathematics Student, vol. 63, no. 1-4, pp. 123-145, 1994. 
[4] S. D. Flåm and A. S. Antipin, "Equilibrium programming using proximal-like algorithms," Mathematical Programming, vol. 78, pp. 29-41, 1997.

[5] S. Takahashi and W. Takahashi, "Viscosity approximation methods for equilibrium problems and fixed point problems in Hilbert spaces," Journal of Mathematical Analysis and Applications, vol. 331, no. 1, pp. 506-515, 2007.

[6] P. Hartman and G. Stampacchia, "On some non-linear elliptic differential-functional equations," Acta Mathematica, vol. 115, pp. 271-310, 1966.

[7] J.C. Yao and O. Chadli, "Pseudomonotone complementarity problems and variational inequalities," in Handbook of Generalized Convexity and Generalized Monotonicity, J. P. Crouzeix, N. Haddjissas, and S. Schaible, Eds., vol. 76, pp. 501-558, Springer, New York, NY, USA, 2005.

[8] H. Zegeye, E. U. Ofoedu, and N. Shahzad, "Convergence theorems for equilibrium problem, variational inequality problem and countably infinite relatively quasi-nonexpansive mappings," Applied Mathematics and Computation, vol. 216, no. 12, pp. 3439-3449, 2010.

[9] H. Zegeye and N. Shahzad, "A hybrid approximation method for equilibrium, variational inequality and fixed point problems," Nonlinear Analysis. Hybrid Systems, vol. 4, no. 4, pp. 619-630, 2010.

[10] L. C. Zeng, S. Schaible, and J. C. Yao, "Iterative algorithm for generalized set-valued strongly nonlinear mixed variational-like inequalities," Journal of Optimization Theory and Applications, vol. 124, no. 3, pp. 725-738, 2005.

[11] X. Qin, Y. J. Cho, and S. M. Kang, "Viscosity approximation methods for generalized equilibrium problems and fixed point problems with applications," Nonlinear Analysis. Theory, Methods $\mathcal{E}$ Applications, vol. 72, no. 1, pp. 99-112, 2010.

[12] X. Gao and Y. Guo, "Strong convergence of a modified iterative algorithm for mixed-equilibrium problems in Hilbert spaces," Journal of Inequalities and Applications, vol. 2008, Article ID 454181, 23 pages, 2008.

[13] C. Jaiboon and P. Kumam, "A hybrid extragradient viscosity approximation method for solving equilibrium problems and fixed point problems of infinitely many nonexpansive mappings," Fixed Point Theory and Applications, vol. 2009, Article ID 374815, 32 pages, 2009.

[14] C. Jaiboon and P. Kumam, "Strong convergence for generalized equilibrium problems, fixed point problems and relaxed cocoercive variational inequalities," Journal of Inequalities and Applications, vol. 2010, Article ID 728028, 43 pages, 2010.

[15] C. Jaiboon, P. Kumam, and U. W. Humphries, "Weak convergence theorem by an extragradient method for variational inequality, equilibrium and fixed point problems," Bulletin of the Malaysian Mathematical Sciences Society, vol. 32, no. 2, pp. 173-185, 2009.

[16] J. S. Jung, "Strong convergence of composite iterative methods for equilibrium problems and fixed point problems," Applied Mathematics and Computation, vol. 213, no. 2, pp. 498-505, 2009.

[17] J. K. Kim, S. Y. Cho, and X. Qin, "Hybrid projection algorithms for generalized equilibrium problems and strictly pseudocontractive mappings," Journal of Inequalities and Applications, vol. 2010, Article ID 312602, 18 pages, 2010.

[18] P. Kumam and C. Jaiboon, "A new hybrid iterative method for mixed equilibrium problems and variational inequality problem for relaxed cocoercive mappings with application to optimization problems," Nonlinear Analysis. Hybrid Systems, vol. 3, no. 4, pp. 510-530, 2009.

[19] S.-Y. Matsushita, K. Nakajo, and W. Takahashi, "Strong convergence theorems obtained by a generalized projections hybrid method for families of mappings in Banach spaces," Nonlinear Analysis. Theory, Methods E Applications, vol. 73, no. 6, pp. 1466-1480, 2010.

[20] J.-W. Peng and J.-C. Yao, "Strong convergence theorems of iterative scheme based on the extragradient method for mixed equilibrium problems and fixed point problems," Mathematical and Computer Modelling, vol. 49, no. 9-10, pp. 1816-1828, 2009.

[21] P. Katchang and P. Kumam, "A general iterative method of fixed points for mixed equilibrium problems and variational inclusion problems," Journal of Inequalities and Applications, vol. 2010, Article ID 370197, 25 pages, 2010.

[22] W. Kumam, C. Jaiboon, P. Kumam, and A. Singta, "A shrinking projection method for generalized mixed equilibrium problems, variational inclusion problems and a finite family of quasinonexpansive mappings," Journal of Inequalities and Applications, vol. 2010, Article ID 458247, 25 pages, 2010.

[23] Y. Yao, Y.-C. Liou, and J.-C. Yao, "A new hybrid iterative algorithm for fixed-point problems, variational inequality problems, and mixed equilibrium problems," Fixed Point Theory and Applications, vol. 2008, Article ID 417089, 15 pages, 2008. 
[24] H. Zegeye and N. Shahzad, "A hybrid scheme for finite families of equilibrium, variational inequality and fixed point problems," Nonlinear Analysis, Theory, Methods and Applications, vol. 74, no. 1, pp. 263272, 2011.

[25] H. Zegeye and N. Shahzad, "Approximating common solution of variationalinequality problems for two monotone mappings in Banach spaces," Optimization Letters. In press.

[26] A. Tada and W. Takahashi, "Weak and strong convergence theorems for a nonexpansive mapping and an equilibrium problem," Journal of Optimization Theory and Applications, vol. 133, no. 3, pp. 359-370, 2007.

[27] X. Qin and S. M. Kang, "Convergence theorems on an iterative method for variational inequality problems and fixed point problems," Bulletin of the Malaysian Mathematical Sciences Society, vol. 33, no. 1, pp. 155-167, 2010.

[28] P. Kumam and C. Jaiboon, "A system of generalized mixed equilibrium problems and fixed point problems for pseudocontractive mappings in Hilbert spaces," Fixed Point Theory and Applications, vol. 2010, Article ID 361512, 33 pages, 2010.

[29] F. E. Browder, "Nonlinear operators and nonlinear equations of evolution in Banach spaces," Proceedings of Symposia in Pure Mathematics, vol. 18, pp. 78-81, 1976.

[30] W. Takahashi, Nonlinear Functional Analysis, Yokohama Publishers, Yokohama, Japan, 2000.

[31] G. L. Acedo and H.-K. Xu, "Iterative methods for strict pseudo-contractions in Hilbert spaces," Nonlinear Analysis. Theory, Methods \& Applications, vol. 67, no. 7, pp. 2258-2271, 2007.

[32] C. Martinez-Yanes and H.-K. Xu, "Strong convergence of the CQ method for fixed point iteration processes," Nonlinear Analysis. Theory, Methods E Applications, vol. 64, no. 11, pp. 2400-2411, 2006.

[33] R. E. Bruck Jr., "Properties of fixed-point sets of nonexpansive mappings in Banach spaces," Transactions of the American Mathematical Society, vol. 179, pp. 251-262, 1973.

[34] J.-W. Peng and J.-C. Yao, “A new hybrid-extragradient method for generalized mixed equilibrium problems, fixed point problems and variational inequality problems," Taiwanese Journal of Mathematics, vol. 12, no. 6, pp. 1401-1432, 2008.

[35] P. L. Combettes, "The convex feasibility problem: in image recovery," in Advances in Imaging and Electron Physics, P. Hawkes, Ed., pp. 155-270, Academic Press, Orlando, Fla, USA, 1996.

[36] T. Kotzer, N. Cohen, and J. Shamir, "Images to ration by a novel method of parallel projection onto constraint sets," Optics Letters, vol. 20, pp. 1172-1174, 1995.

[37] M. I. Sezan and H. Stark, "Application of convex projection theory to image recovery in tomograph and related areas," in Image Recovery: Theory and Application, H. Stark, Ed., pp. 155-270, Academic Press, Orlando, Fla, USA, 1987.

[38] Y. Censor and S. A. Zenios, Parallel Optimization, Numerical Mathematics and Scientific Computation, Oxford University Press, New York, NY, USA, 1997. 